\title{
Turing landscapes
}

\section{Citation}

Ervin, Stephen M. "Turing Landscapes Computational and Algorithmic Design Approaches and Futures in Landscape Architecture." In Codify : Parametric and Computational Design in Landscape Architecture, 89-116. Abingdon, Oxford: Routledge, 2018.

\section{Permanent link}

http://nrs.harvard.edu/urn-3:HUL.InstRepos:39148377

\section{Terms of Use}

This article was downloaded from Harvard University's DASH repository, and is made available under the terms and conditions applicable to Other Posted Material, as set forth at http:// nrs. harvard.edu/urn-3:HUL.InstRepos:dash.current.terms-of-use\#LAA

\section{Share Your Story}

The Harvard community has made this article openly available.

Please share how this access benefits you. Submit a story.

\section{Accessibility}


Computational and algorithmic design approaches in landscape architecture (published in Cantrell, Bradley \& Adam Mekies, Codify: Parametric and Computational Design in Landscape Architecture, Routledge, 2018.)

\section{Introduction}

Landscape architecture and landscape architects may have three rather different, although naturally interconnected, modes of interaction with computation (computing hardware, displays, software, and the many emergent technologies of telecommunications and electromechanical devices, e.g. mobile phones, drones, robots, immersive VR headsets, et al.):

1. Using software - as a tool

2. Writing code - as a language

3. Engaging with the Internet of Things - as a medium

The simplest and historically earliest adopted mode is just using commercial software, from wordand image-processing to CAD, GIS and video-conferencing. The next is writing software, or coding, whether simple macros embedded in other software, or free-standing applications, for informationanalysis (more common) or as part of design idea-generation (emergent). The final mode is engaging with connected and computational devices - the ever-expanding 'Internet-of-Things' (IoT), from sensors to actuators, to new high-tech building materials and devices - in environmental design. The first caused some upheaval in the 1980s and 1990s as rapid development and adoption of software offered new instrumental possibilities to landscape architects - mostly in automation, accuracy and convenience; but entailed no real challenge to conceptions of landscape or even, for most early-adopters, modes of practice, design thinking, or representation. Computer graphics did offer a new and compelling medium for graphic representation, including video, and generated some instrumental and conceptual reevaluation of and invention of new representational conventions by the turn of the century. The second mode, 'writing code', seemed innocuous and mostly instrumental at first, again mostly for automation and convenience, but significantly, also for customization and expressive power, which, once experienced, was captivating for many designers. Today, code is a recognized medium alongside sketchbooks and scale models, and understood to contain rich design potential which has only barely begun to be tapped. The third - engaging with computation via the loT, not just as aides to conceiving of or representing landscapes composed of the traditional palette of landform, plants, water, and structures, but as actual embedded components of new kinds of landscapes, especially 'interactive' and 'responsive' ones - is the most radical mode of interaction with computation for landscape architects. Coexisting with and often dependent upon custom coding, this form of 'computation-aided-design' (not just 'CAD') entails the greatest challenge to conceptions of landscape, of interaction, and of design process and representation.

Today, designing with algorithmic approaches and computational tools and embedding electromechanical computer-controlled devices in the landscape are becoming commonplace. Since all these approaches are enabled by computers operating on basic principles now known as 'Turing Machines', after the inventor and theorist Alan Turing, I call the landscapes and environments so imagined and created 'Turing Landscapes'.

Consider a simple $2 \times 2$ matrix: Landscapes designed with and without computation $\mathrm{X}$ landscapes with and without embedded computation (digital/electromechanical elements in addition to natural / physical elements). 


\begin{tabular}{|c|l|}
\hline $\begin{array}{l}\text { Landscapes designed } \\
\text { without computation, } \\
\text { but with } \\
\text { embedded computation }\end{array}$ & $\begin{array}{l}\text { Landscapes designed } \\
\text { with computation, } \\
\text { and with } \\
\text { embedded computation } \\
\text { (Turing landscapes) }\end{array}$ \\
\hline rare / simple \\
$\begin{array}{l}\text { Landscapes designed } \\
\text { without computation, } \\
\text { and without } \\
\text { embedded computation } \\
\text { (Oimsted landscapes) }\end{array}$ & $\begin{array}{l}\text { Landscapes designed } \\
\text { with computation, } \\
\text { but without } \\
\text { embedded computation }\end{array}$ \\
\hline pre- year 1990 & \\
\hline
\end{tabular}

Landscapes designed without computation and without embedded computation (call them 'Olmsted landscapes') are very familiar - the vast majority of landscapes designed and built before the year 1990. These traditional landscapes are composed of six essential elements: landform, vegetation, water, infrastructure (including buildings), animals (including people), and atmosphere (including lighting) [ERVIN]. Since the advent of sufficiently powerful commercially available software on affordable personal computers, landscapes designed (and, increasingly, built) with computation of some sort are now the vast majority. Landscapes with embedded computation - that is, not just made of plants, paving, and structures, etc., but also containing some forms of sensors, processors, and electromechanical actuators controlled by code - are still rare, or have only primitive computation embedded (increasingly many public and private landscapes have computer-based irrigation or lighting controllers embedded, for example). Landscapes designed with computation, and with embedded computation - call them 'Turing landscapes' - are emergent, but still uncommon. A computer generated landscape scene experienced through completely simulated Virtual Reality (VR) or overlaid real/simulated Augmented Reality (AR) is one example of a Turing landscape (the VR headset counts as the embedded computation - even though some might argue that it's not truly a 'landscape', a semantic distinction I won't contest). A modern public park, designed with GIS and CAD software, and possibly custom coding, and containing embedded sensors, WiFi channels delivering custom geo-specific information to visitors, actuators controlling light, water, shade, sound and other environmental qualities, and other robotic computer-controlled entities is more fully a Turing landscape - and may well become the norm in this $21^{\text {st }}$ century.

Since even before the microcomputer revolution of the late 1970s - and certainly ever since then - the design community has struggled with questions around the nature, value, and implications of 'computer-aided design'. Early CAD software, originally designed to enable the production of precise and repetitive drawings, focused much of this discussion on representation technologies, and automation, and then on questions of creativity, and the perceived lack thereof in computerized processes. At the same time, research and developments in Artificial Intelligence (AI) and robotics fueled a debate about humanversus machine-like attributes, sometimes heated and ideological, and that sometimes seemed to fit into a larger culture-clash between the arts and humanities on the one hand, and science, engineering and technology on the other. In landscape architecture and design, always somewhat uncomfortably straddling art and science, Leo Marx's 1964 "Machine in the Garden" [MARX] trope was reflected as a widespread discomfort with technology in design processes, an expressed preference for 'soft pencil sketches on toothy paper' as evidence of real creativity, and a dismissal of computation as suitable only for numerical calculations and repetitive operations.

Almost all the digital computers we are familiar with using work on some basic principles first laid out in the 1930s and 40s, by theorists and inventors John von Neumann and Alan Turing, who, separately and together, described in outline a machine capable of taking in inputs and storing and retrieving values (together 'data'), controlled by commands and operations (we might call them 'programs and subprograms') capable of producing outputs, as a result of arbitrarily complex calculations, or procedures ('algorithms'). Their work was both abstract and theoretical to begin with, and also practical, as they built early prototypes of modern digital computers. [TURING, VON NEUMANN] Together they early on recognized 
the implications of such machines as being capable of reasoning, not just calculation; and articulated many of the earliest ideas and questions about 'artificial intelligence'. Today, Information and Communications Technology (ICT) is pervasive, and includes mobile devices, drones, sensors and robotic actuators, among many other technologies.

In 1983, in the seminal text Microcomputers in Landscape Architecture [MAcDougALL] the chapter on 'Computer Applications for Landscape Architects' opened with the category: 'Word Processing'. This simple fact that the typewriter could be replaced - with great benefit, by a computer, word-processing software, and printer - really was the beginning of the microcomputer revolution. The very next 'killer app' for most professionals, beyond word-processing, was 'number-crunching', with the proto-spreadsheet software 'VisiCalc'. That computation and ICT might be more than just instrumental amplifiers of human eyes, hands \& brains (supporting 'techne), but might indeed be media for exploration and expression of ideas and abstract concepts (supporting 'poesis); might like other media bring their own characteristics, constraints, and affordances to design processes; and might, like other fields and disciplines, bring their own ways of knowing, ways of doing, and aesthetics, potentially amenable to fertile hybridization, were not initially obvious, and still are not particularly mainstream ideas (but increasingly so, as this volume demonstrates! ) Whether they are analog landscapes meant to built and visited or appreciated in situ, or virtual landscapes meant only to be viewed/experienced through audio-visual (and perhaps tactile/kinetic) devices (e.g. 'VR goggles'), is not a critical distinction; they are both designed, and both invite spatial/sensory experience and appreciation. Perhaps the earliest example of a computer-generated virtual landscape was "The Road to Pt Reyes", a 1983 composition by Alvy Ray Smith and others, [SMITH] that featured algorithmic methods to create terrain, plants, road surface materials, reflections, atmospheric haze and even a rainbow. (Figure 1.)

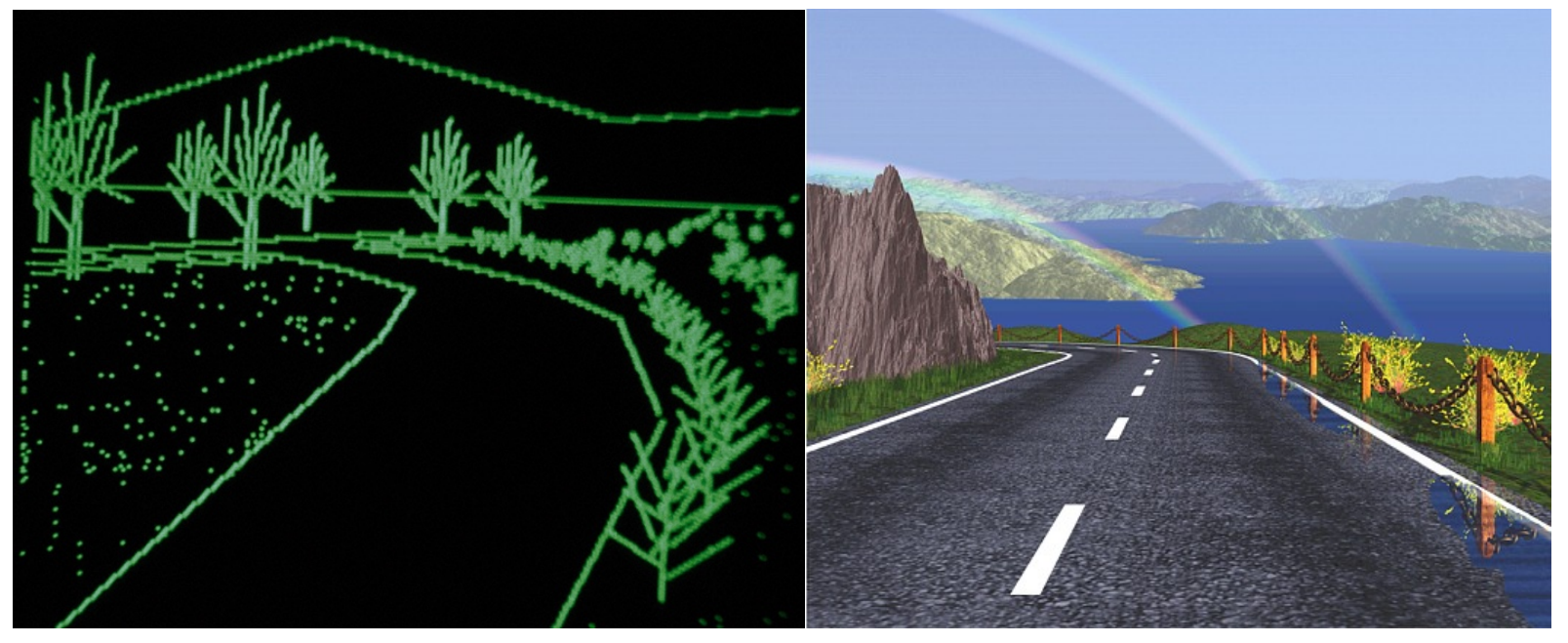

Figure 1. Early computer-generated virtual landscapes: (a) "Valley Road", Stephen Ervin 1983; (b) "The Road to Pt Reyes", composition by Alvy Ray Smith et al. 1983 (c) PIXAR. Used with permission.

\section{Computer Aided Landscape Architectural Design}

Definitions of landscape architecture are necessarily broad. With origins in both the natural sciences, problem solving, and engineering on the one hand, and visionary imagination, social psychology, and aesthetic composition on the other, the discipline spans many scales and concerns - but all are about design, and about environments, with an emphasis on living and dynamic ones. The tools of the trade are equally diverse, and include notebooks, cameras, computers, and shovels - but many of them are primarily concerned with representation, since the design and management of environments are often done with surrogate representations, rather than with full-scale living realities, for a mix of convenience, practicality, safety and ethical reasons.

As with any art, or field of human endeavor, a specialized language is also employed. The 
relationships between art, science, and language are deeply intertwined, and philosophically rich, but suffice it to say that manipulating sounds or signs (symbols, letters, numbers, say) as a way of expressing meaning, or emotion, is fundamental to both art and science; and part and parcel of design and of problem solving. Designs and design acts may then be understood as assertions, answers to questions (often more exploratory than definitive) and the language used - whether drawings of circle and arrows, or actual rows of actual trees, or words or equations - certainly have an intimate relationship to, and often a formative influence on, both the creation and experience of the design or designed artifact or process.

Computer Aided Design (CAD) in its original conception was seen as a way to assist designers with graphic symbol manipulation - drawing circles, lines, and arrows more precisely, or more rapidly, or with effortless repetition, rotation and scaling, etc. The very first instance of CAD software, written in 1963 by Ivan Sutherland at MIT [SUTHERLAND] was called 'Sketchpad', with a nod to the essential role of sketches and sketchpads for making notes, drawings, and diagrams in design processes. Tellingly, the modern Java-based Processing language [PROCESSING] calls stored programs 'sketches'. This idea that notations in some language (e.g. graphite on paper, or programs in Java) are essential to design underlies the role and value of computation in landscape architecture.

There are other more immediate/instrumental applications of computation that have led to its general applicability and adoption by so many disciplines and activities, such as speed, accuracy, portability, etc. Some of the earliest computer software introduced to landscape architects was from civil engineering, for such calculations as hydrologic runoff, cut-and-fill mass-balance calculations, and coordinate geometry for horizontal and vertical road alignment. The recent emergence of the landscape architectural practice known as 'geodesign' [STEINITZ] is based on a combination of computational approaches: GIS-based mapping, spatial analysis, and cartographic representation software, CAD drawing and design tools, simulation-based impact analyses (such as cost, connectivity, biodiversity, energy efficiency, etc.), and on-line web-based tools for group collaboration, documentation, and videoconferencing, designed to enable groups of professionals, scientists, engineers, stake-holders and citizens to collaborate on large problems over extended distance and time, with multiple perspectives and criteria. This development, enabled by satellites, powerful computers, fast networks and other ICT tools represents a much more expansive definition of 'computer aided design' than 'drawing tool' - even though, at its core, it is still just symbol manipulation.

\section{Computation - as technology, and as a medium for design}

Many people think of computers as having to do with mathematics, and it is true that many of the earliest practical practical applications of computation were for calculation, and many still are today. But at their core, computers are much more about logic than they are about math. Alan Turing and John Von Neumann, the real inventors of most modern day computing, were both motivated by the need for rapid and repetitive calculations during the second World War; but both were drawn immediately into broader and more abstract considerations about 'thinking' machines and the nature of human knowledge; both were envisioning 'intelligent machines', and the fact that the essential electronic storage was called 'memory' was a distinct reference to the characteristics of the human brain.

The principle insight that Turing had was that a suitably designed machine could be 'general purpose' rather than being designed just to do one thing - say, add two numbers - and this meant that the sequence of instructions about what to do - the 'program' - had to be 'data', capable of being input to the machine, just like other data such as the numbers to be added together. Thus, although a digital computer could be an adding machine - and the first hand held popular computers were indeed calculators, that could perform many other mathematical operations than just addition - it could equally well be a text formatting machine, or a music generating, or a drawing display machine, etc.

An essential characteristic of Turing's universal computing machine design was that processing steps such as entering, adding, displaying, etc. had to be sequential, one at a time. This reflected both the limitations of electro-mechanical equipment at the time, and also the basic human notion of a process, or recipe, as a sequence of steps. Although branching and repetition were immediately understood to be essential for many real tasks, they were nonetheless always in sequential time, one-at-a-time. Turing's claim was that a single universal machine could be built to perform any process of this sort - and which 
he called an algorithm, using a word first used by a $6^{\text {th }}$ century Persian mathematician. So pervasive is this idea that computational approaches are often called 'algorithmic' approaches, and so we often hear of computer-enabled design processes, enabled by digital computers (that is, truly 'computer-aided design') referred to as 'algorithmic design'.

Modern day computer electronics have made possible machines that can do more than one thing at a time, simultaneously; these are usually called 'parallel processors'. While this inherently makes for faster and more powerful computation, it turns out that algorithms for these non-Turing computers must be noticeably different from those for simple one-step-at-a-time sequential processes. Indeed, a whole world of complications opens up with parallel processing, and while this is a major study in computer science, suffice it to say that few algorithmic approaches in landscape architecture have yet been influenced by the demands or capabilities of parallel processing. (One possible exception - the use of socalled 'cellular automata' to simulate landscapes and landscape processes, is discussed below. Otherwise, no further mention of parallel processing will be made.)

All modern digital computers and computation have one more salient aspect; they are built on binary representations, and they employ boolean logic, named after the mathematician George Boole who formalized the ideas of the fundamental binary values TRUE and FALSE, represented as 1 and 0 respectively, and logical operators, such as AND, OR, NOT and others. In fact, even for mathematical calculations all numbers are represented in binary (just as letters, and colors, and other symbols are), and even processes such as multiplication or drawing a curved line are encoded as algorithms involving simple logical operations on individual binary digits, or bits. The process of digitization - taking measurements in the real, analog world and converting them to bits or pixels (such as a digital camera does) - is fundamental to digital computation, and can give rise to both scientific concerns about accuracy and precision on the one hand, and unintended and intentional aesthetic effects on the other; both are explicit in computational approaches in landscape architecture. (For example: the raster cell size of satellite imagery may affect landscape ecological analysis of patterns and processes; and 32-bit vs 8bit vs 1-bit color makes a huge difference in digital images' appearance.)

Building on the foundation of these binary building blocks, the art and science of expressing algorithms - or 'writing code' - has evolved substantially in the nearly 70 years since the first digital computers, when programs needed to specify every individual logical operation on nearly every individual bit of data, in extremely granular detail in highly specialized code sometimes called 'machine language'. Today, much more high-level aggregations can be expressed in simple code, so that a modern landscape architect could write something like:

(Draw-row-of-trees type=maple path $=($ Get-road-segment start $=\mathrm{STA} 1+50$ end $=\mathrm{STA} 2+50)$ alignment=parallel

offset $=10$ spacing $=12$ number $=9$ )

; 10m safety regulation

;; $12 \mathrm{~m}$ arborists recommendation

;" design decision

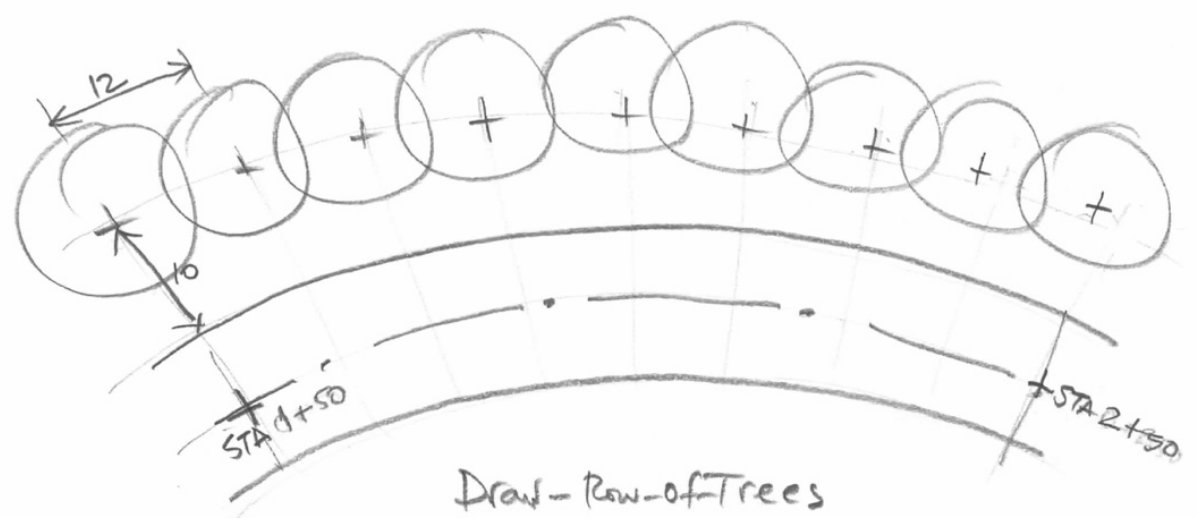

Figure 2. Draw-row-of-trees() output 
This snippet of made-up code shows many of the characteristics of modern high-level programming languages, particularly those called 'imperative' and 'procedural' languages: named variables, named procedures with specific required input parameters (e.g. Draw-row-of-trees... and Getroad-segment...), access to libraries and databases of values, and plain English comments to clarify the steps in a process, or the reasons for particular values or calculations. All common programming languages also usually have some ways of expressing repetition (number of times, or until some condition is reached), and conditional tests (IF-THEN statements. (Although not seen directly in this snippet, the draw-row-of-trees procedure will likely use some form of repetition, taking the input parameter number $=20$ into account.)

The usual minimum functionality of any programming language includes:

- Some means of taking input, e.g. letters from a keyboard, or points from a touchscreen, etc.

- Stored variables with names and values, often of different types (integers, strings, arrays, etc.)

- Stored procedures (or subroutines, or methods, etc.) that take inputs and return values or produce outputs

- A range of operations on variables, such as logical, mathematical, string-manipulation, etc.

- Some means of managing repetition (e.g. number of times, or until some condition is reached)

- Some means of conditional branching of control (e.g. testing for true/false, or some condition)

These six simple elements (and usually some other useful programming language functions) can be combined into endless variety and permutation, including every CAD program, GIS program, imageprocessing and speech-recognition program, web service and mobile phone app, etc. Algorithms are usually written to serve some particular processing purpose, and then implemented in programs with inputs, outputs, error checking, interfaces, and other usability features. By changing one or more values, say 'size or 'spacing, many different variations can be tried out and evaluated in the design process.

Thus the act of writing code is both a technical exercise in appropriate use of specified syntax, and a design activity, involving intentions, choices, aesthetics, and ramifications. In this way it is quite similar to making a construction drawing, or a specification sheet; coding provides yet another language for expressing and exploring design intent in the creative and production processes of imagining and creating landscapes. Designing procedures and sub-procedures that deal with one specialized task, and communicate with procedures - the process of 'modular decomposition' [ALEXANDER] is akin to breaking a large problem into smaller problems that can be combined together, or even analogous to the processes in construction of various subcontractors working on different but connected parts of a project. In this way, too, writing code is a design exercise: identifying and naming major procedural modules and specifying their interconnections, has direct parallels with spatial and formal design decisions.

When writing code, one must make choices about what is input and what is output (in scientific terms, independent and dependent variables), which is often an important step in determining 'what can the designer control?', and what are the most important, or effective, control variables. Design, of course, often involves changing these around (e.g. "Should the length of the road determine the number of trees? Or the number of trees determine the length of the road? Or will the budget determine both? ") Writing code that can take various inputs, and produce outputs that can then be compared and evaluated, is an increasingly useful way of exploring these alternatives - a design process.

Exploring alternatives is a common and essential design activity, and highlights an important point: "What exactly do we mean by exploring?" Exploration is not usually an algorithm, because unforeseen conditions and demands may arise - we can never write down every possible eventuality in an IF-THEN rule in advance. So exploration is more akin to a 'heuristic' - a general approach, setting out initial steps and general principles, but also leaving open the chance of discoveries and opportunities. In this kind of computer-aided design, we most often find a human being, exploring in partnership with a computer program. The program may be able to generate alternatives, and even partially evaluate them, but the 'flashes of insight' or 'inspiration' we often associate with successful design are difficult to explain, much less to program into an algorithm. (Various attempts have been made, over several decades of artificial-intelligence research; but none are completely convincing, and the Al questions are deep, and so we leave this topic out of the rest this discussion.) 
Coding in support of design exploration can be understood as a process of:

- Identifying key design aspects, or measurements or characteristics of concern, and giving them names so they can be manipulated as variables;

- Identifying key relationships between design variables, that can be computed, perhaps in a simple formula, perhaps in a more complex multi-step algorithm;

- Creating procedures (algorithms) to encapsulate necessary or desirable processes (such as 'calculate stair dimensions from topographic rise and materials choice', or 'compute change in biodiversity in $30 \mathrm{~m}$ square cells for the entire study area', or 'evaluate likely visual experience given new road alignment', etc.)

- Generating outputs - numeric, textual, graphic, sound, video, 3D, etc - for human evaluation, or for communication or construction.

The last step in this outline characterizes the model of design proposed by Herbert Simon in 1969, called simply 'Generate and Test' [SIMON]. In this view, two distinct aspects of design thinking 'synthesis' and 'analysis' - can be considered (and programmed). Which or how much of each (generating, or testing) is done by human or computer hardly matters. Perhaps computation can be used to do the heavy math of a fluid dynamics calculation to evaluate (test) the airflow around a proposed (generated) sculptural shape; or perhaps an algorithm can be used to generate shapes and patterns for evaluation (testing) by the human designer's eye. In this dance between 'generate' and 'test', there is a continuous feed-back loop of iteration and refinement, ultimately leading to some 'final' design decision, based on a heuristic approach, not specified by any algorithm.

Some have wondered how any predictable sequence of operations could give rise to invention, or design; and there are many questions about artificial intelligence, or artificial creativity, and whether any digital computer is even theoretically capable of intelligence or design creativity. But that is not necessary for computer-aided design, in which a human partner provide the design intelligence - at least for now. One approach to escaping the limitations of predictable algorithms has been to turn to randomness injecting both random numbers and random logical decisions into algorithms, to generate graphics, say. In general, complete randomness is never satisfactory - it just creates white noise; but 'controlled randomness' within some ordered process can give rise to variation that adds interest and diversity. Some computer scientists have explored an approach to programming called genetic algorithms, that use random processes, akin to genetic mutation, to alter code small steps at a time, using multiple repeated trials and generations to ultimately make code that is artificially 'evolved' to be well suited to some task or goal. Finding applications of genetic programming in landscape architecture is not easy, but the potential for interesting results is there.

Given the capabilities and limitations of computation as discussed, there are three distinct ways, or modes, in which algorithmic/computational approaches are commonly used and valuable to designers:

1. As Tool: Using out-of-the-box commercial software to create graphics, various analyses, and other media/artifacts, used both to 'generate' and to 'test';

2. As Language: Writing code, in some programming environment, to generate graphics or other output, used both to 'generate' and to 'test';

3. As Medium: Using computational tools \& code to receive input from the physical world (in real-time, via sensors) and/or directly control something (display, sound, electromechanical actuator, etc.) in the physical world.

3.1. Out-of-the-box commercial software as tools for helping to create drawings, renderings, calculations, simulations, analyses, etc. These tools use underlying algorithmic techniques, but these are not exposed, usually little knowledge of them is required to use the software, and in general the software cannot be modified from its predicted routine and capabilities, as powerful as they may be. This 'using software' mode - the simplest -helps with the convenience and productivity of drawings for the purposes of design - and is by far the most common. To the extent that CAD, GIS and Image-processing software comprise a multi-purpose tool-box, they can be appropriated and used by designers in a wide range of ways. For landscape designers, these are mostly to make graphic representations, 3D models, videos, and construction documents that describe and prescribe physical interventions in the landscape to be made by people and machines. They may of course also be employed to create only 'virtual landscapes', 
to be viewed via VR technology, mostly for entertainment but also for artistic and other valid purposes.
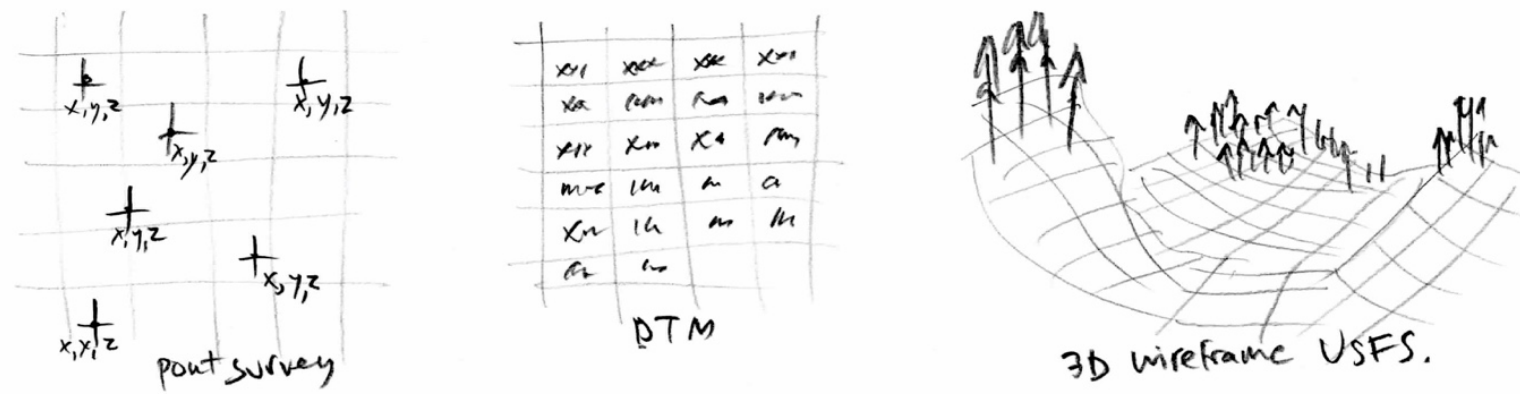

Figure 3. AS TOOL : From Measurement to Data Structure to Graphics

3.2. 'Writing code', either in the context of some graphics or other software, perhaps leveraging an Application Programmers Interface (API) to invoke and control built-in functions of the software; or in a general-purpose programming language (C++, or Python, or Javascript, et al.), connected to some hardware capabilities, such as generating graphics, or text reports, or web content, etc. Custom-built code, especially by an amateur, may be more difficult to produce or less effective in performing complex routines for which commercial software has already been perfected; but may also be the only way to get exactly the effect desired, if that effect is not included in any commercial software, and it may be more convenient to explore and experiment with, in that regard. Writing code is less common than using software among landscape architects (indeed, most people), but is becoming increasingly so, as some working knowledge of coding, in some language, is becoming part of everyday training for design professionals. In the US, many high school students now learn coding in some form (there are even proposals to allow learning to code to fulfill a 'foreign language' requirement!) Consequently, landscape architects and designers in post-secondary education and graduate-school are increasingly code-savvy, and able to incorporate coding as part of the creative and production processes. The proliferation of webbased apps and mash-ups has made the idea of accessible, often custom-built, code-based tools for information-based design purposes a normal expectation of a whole generation of designers.

This 'writing code' - requiring different training and knowledge than simply 'using software' - can be used to directly express abstract design ideas, and is often used to produce artifacts (graphics, say) that can stand alone or be imported into commercial software for production purposes. The 'Processing' [PROCESSING] language (built on Java), designed especially for artistic and graphics coding, is one effective approach, which has ease of entry, thanks to its clever packaging of essential graphics functions into simple commands, as well as deep expandability, since it is based on the robust and full-featured Java language. Another popular approach is using graphical user interfaces (GUIs) to CAD software, such as 'Rhinoceros' and 'Grasshopper' [MCNEEL] which enables the construction of code by graphical programming, dragging arrows between boxes representing logical/mathematical functions and built-in capabilities of the underlying Rhino software. Landscapes created by code can be quite abstract, using only mathematical and other parameters determined by the programmer, or through some interactive user interface; or they may be responsive to site information, or other base data, such as terrain read-in as a series of elevations, or contour lines, etc. Virtual landscapes created by code can be designed to mimic and explore natural processes and forms, such as branching, erosion, flow, etc.; or can be quite fantastic, not meant to reflect any natural systems, or even to be built; but nonetheless to be spatial, and to elicit human response, even if only through VR or AR headsets.

An important aspect of writing code for any purpose is to match algorithms with data structures. Certain algorithms work best with regularly spaced grids of values; others with linked tables, others with text strings, and so on. Sources and methods of capturing digital data (digitizing) directly influence this choice: digital cameras and satellite sensors generate raster grids of values; field surveying units lists of $(\mathrm{x}, \mathrm{y}, \mathrm{z})$ values; hand sketches may be transcribed as 2D lines and curves. Design algorithms link these inner representations, held in computer memory and operated upon by programming language primitives, with outer representations: 2D line drawings, color raster prints and renderings, 3D fabricated models, and others. The fundamental landscape medium of topography can be described and manipulated as a 
collection of 3D points (a point cloud); a regular grid of z-values (digital terrain model); a set of curved lines with associated elevation attributes (contour lines); a linked collection of 3D triangles (triangulated irregular network); in some cases a mathematical equation, or collection of trigonometric functions (e.g. $x^{\wedge} 2+y^{\wedge} 2+z^{\wedge} 2 / 2<8$ ); and even a descriptive phrase (e.g. "hemispherical mound $8 m$ in diameter, $4 m$ tall") . Each of these has its best uses and limitations, some set by requirements of construction; and as with poetry and foreign languages, translations between one form of representation (data structure) and another is not always exact. Mismatches introduced by translation may be seen only errors or undesirable artifacts; or they may become opportunistic design features, appropriated for some aesthetic or abstract purpose, in the crucible of design ideation.

In common procedural programming languages, many individual values are usually involved, and may have names such as x1, x2, width, color, type1, type2, type3, etc. An essential part of the art of coding is using variable names that are descriptive, and keeping track of the role that each variable plays in an overall system or algorithm. These variables are typically highly granular, and though they may be used in a collection to represent some physical object, often the overall object is never itself named or individually handled. In 'object-oriented programming', however, the initial emphasis is on creating and naming software 'objects' that represent real-world-objects, and that are characterized by having a set of attributes, and behaviors, that are all bundled together. Thus a 'tree' may have a species, height, width, age, growth-rate, and condition, and variable names such as tree1.height, tree2.species, etc. In this way systems, landscapes, environments and assemblies can be simulated in software, with a very direct correlation between variable names, values, and actual attributes.

In addition, object-oriented systems may often implement a form of class-object attribute inheritance hierarchy, which can simplify coding and give rise to powerful behavioral performance of systems. For example, a tree class may be created with attributes as above, and then 'maple-tree' object created as a 'kind' of tree, with an additional attribute 'sap-production'. When reasoning about or computing with the maple-tree1, the code can know that all trees have attribute 'height', and in an addition this maple-tree-1 also has a 'sap-production' attribute. The kind of proliferation of objects with special attributes (e.g. even magical powers as found in many video games), is an example of the expressive and flexible nature of this kind of object-oriented programming.

Whether through procedural- or object-oriented- programming, a common use of both commercial software and custom-coding is for the purposes of analysis and simulation. Designers often need to try to predict future consequences of design proposals and alternatives. The most common form, of course, is visual simulation, which seeks to answer the qualitative question "What might some change look like?" (from different points of view, in different seasons, in motion, etc.) Visual analysis can be used for more quantitative answers, such as "Can this location be seen from some other location?" or "What percentage of the sky is visible from the plaza?". But simulations of many other kinds of systems and impacts, not just visual, are equally important. Many social and physical phenomena can be simulated, given sufficient scientific understanding of the mechanisms, and sufficient inputs. Computational Fluid Dynamics (CFD) software such as ANSYS Fluent [ANSYS] can be used to simulate the flow of fluids (e.g. air, water, smoke) around and through 3D environments, and subject to gravity, evaporation, turbulence, and other dynamic changes, and can be used to model the flow of water in a channel or air pollution in a city, given a suitable 3D model to work with. Human behavioral and econometric simulations can model the flow of pedestrians a plaza, or commuter choices in a multi-model transportation system. These simulations can return a range of kinds of values, from numerical summaries to full-color animations, and while mostly designed for the 'testing' side of computer-aided design, are increasingly being used also as a form of inspiration on the 'generation' side as well. 

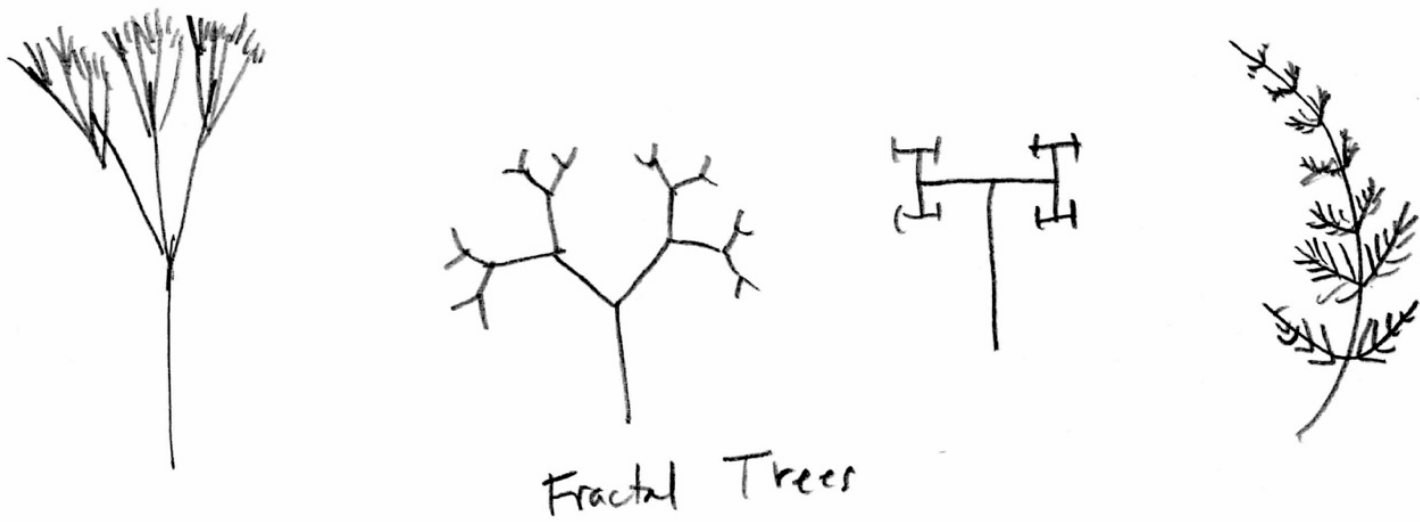

Figure 4. AS LANGUAGE : From Logic to Code to Forms

3.3. Beyond just producing graphics and artifacts for aesthetic or production purposes, and mathematical and other values via simulation, algorithmic approaches can also be used to gather information from the world, through sensors and 'analog-to-digital' converters, expanding the modes of human perception to a wider range of spectra (e.g. infrared) and dimensions (e.g. air or water pollution). Landscape planners and architects have actively embraced the use of a range of remote-sensing technologies, from space satellites with multi-spectral scanners generating raster images of land-cover and weather, to hand-held LIDAR scanners and un-manned-aerial-vehicles (UAVs) or 'drones', creating 3D point clouds representing terrain, vegetation, and built structures. Special purpose software may be needed to collect and decode these data sets, but commercial CAD, GIS and Image-Processing software supply much basic and advanced functionality. The opportunities for custom coding to make creative design use of these sensors and sensed data are many and varied.

For example, using location-based technology embedded in mobile phones or other devices to create web-based-adjuncts to physical landscapes, and providing additional information - either for entertainment, or education - to visitors depending on their location is an example of sensor-based computation-augmented landscape architecture becoming familiar, much like the audio-guides routinely deployed in art museums. In virtual landscapes, such sensors may themselves be virtual - such as proximity sensors in VR interaction environments, which respond to the presence of the human operator's virtual avatar in the virtual landscape.

Finally, beyond sensing and reacting to the physical world, algorithmic tools can also be used to directly control the physical world, by being connected through 'digital-to-analog' converters, to control switches, motors, valves, lights, and other electrical appliances. Motion sensors that automatically open doors sensing an approaching person or controlling water valves in fountains to generate certain choreographic effects are perhaps the most common form of this 'digital sensing and control' mode ordinarily experienced, although more and more are becoming familiar, such as controlling lights for functional or decorative purposes, 3D fabrication tools, robotic earth-moving equipment, computercontrolled flood-gates, or other large-scale artifacts. The loT world of connected 'smart' objects (thermostats, light-bulbs, televisions, houses, cars, cities...) is more fully populated with new devices and new ways of interaction, every day. The Arduino, LittleBits, and Particle [ARDUINO, LITTLEBITS, PARTICLE] family of do-it-yourself loT-tinkerers' hardware kits, including computer controlled motors, lights, and other actuators, as well as sensors, timers, wireless receivers and a host of other interactive components have opened up a whole world of analog-to-digital and digital-to-analog experiments and artifacts, increasingly evident in science labs, art studios, landscape architecture offices, and digital / interactive landscapes and city-scapes.

This final mode, requiring the deepest and most-varied knowledge of digital tools and engineering, is the most complex of the three modes described, and also the most powerful, promising, innovative, and still unfamiliar. 

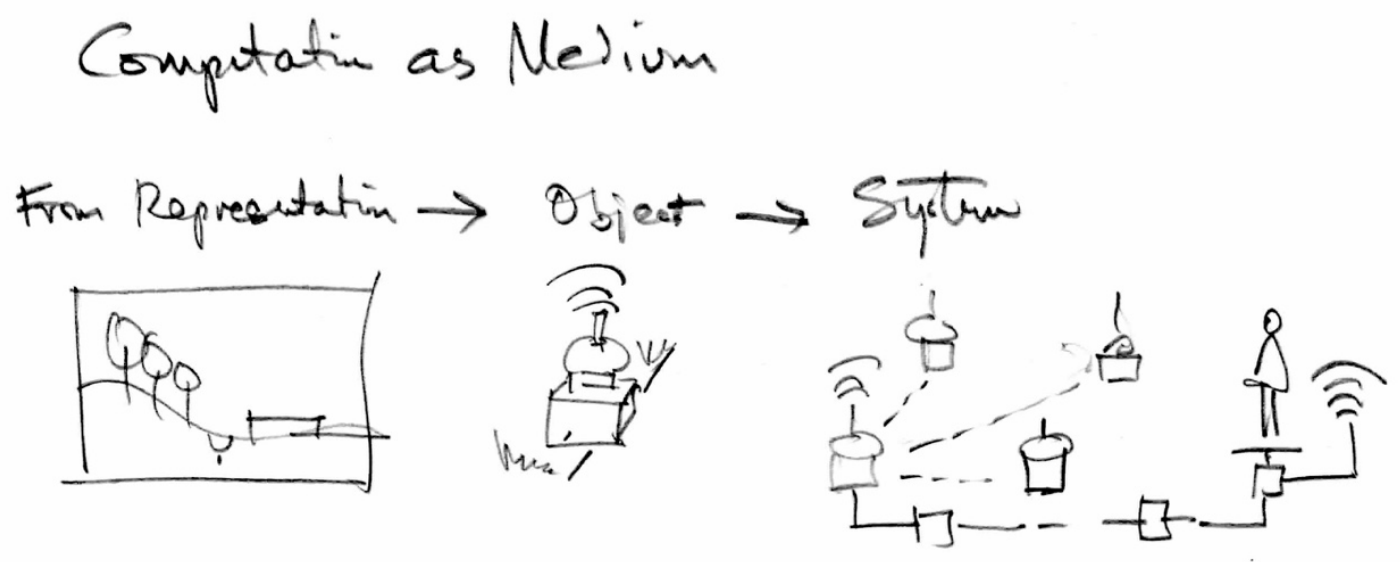

Figure 5. AS MEDIUM : From Representation to Object to System

These three modes - 'using software', 'writing code', and 'digital sensing and control' encompass a broad range of uses and possibilities in design and landscape architecture, both in analog and virtual manifestation.

A special characteristic of landscape architecture, and demand upon landscape design, is the dynamic changing nature of the real world landscape. Under the influence of ever changing weather and climate, landscapes and plants grow and die; water flows, wind blows, the sun rises and sets, and people move through landscapes, and cities and buildings ... Representing these dynamic processes in drawings and still images has long been a challenge - only the very best painters and artists can draw the wind. But with algorithmic tools, and computer-assistance, we can make models of landscapes and landscape elements that capture dynamics and changing states, and can create moving images (video) that represent the dynamics directly. A great power of computational and algorithmic design for landscape architects is this ability to represent, explore, and even design with dynamics. These dynamic landscape animations can represent three fundamentally different points of view:

- Motion through landscapes;

- $\quad$ Motion of landscapes/landscape elements; and

- $\quad$ Interaction with landscapes/landscape elements.

Motion through: The prevalence of computer-generated 'walk-throughs' in architecture and landscape architecture speaks to the central concern with the visual experience of a viewer, or camera, moving along a path taking in a scene. The choreography of visitor experience is an important aspect of landscape and architectural design, and the generation of video animations through a park, landscape, or building is a familiar application of computational tools in landscape architecture. For the most part these are done with commercial software and built-in functions - few designers code their own viewing or camera animation algorithms - though the effects of doing so can be quite dramatic. 'Virtual reality' visualization tools, that immersively engage the visual field, presenting true stereoscopic perspective and responding to head movements and other controls to change views and simulate motion, have for several decades been the domain of video-gamers and some adventurous designers. These are mostly used in the 'test' mode, rather than 'generate'; but the potential for the latter is intriguing.

Motion of: Landscapes and public spaces, of course, are not just static constructions with viewers moving through them (as the simpler walk-throughs would have it!); wind ruffles leaves and blows clouds across the sky, blocking the sun, sometimes bringing rain, and other people in the scene are moving, too... So, both for more realism, and for engaging more expressive components of landscape, digital designs can also be made that imbue motion, as of people walking, wind blowing, rivers flooding, lights moving, etc. These visual simulations are necessarily substantially more complicated than the simpler walk-throughs, and again are mostly made using off-the-shelf software and their built-in dynamics, motion, and animation functions. Many have built-in support for physical simulation of material properties such as elasticity and rigidity that can affect computer generated motion. An enormous and powerful set 
of tools for computer graphics and image rendering, so well developed by the movie special-effects industry, is available to landscape architects today for visual simulations and design explorations.

Interaction with: Finally, people inhabiting or visiting a landscape work not only through a camera lens, viewing motion; they can directly interact with, and influence, the landscape and landscape elements. Whether through touch screens and VR goggles, interacting with a digital model, or via sensors interacting with the real world, the potential for engaging user interaction - beyond just viewing - is one of the most powerful potentials for computer-aided design in the landscape. Working with real landscape elements and digital models of landscape elements that respond to interaction, and algorithms that model behavior and not just dynamics but reactions and interactions - creating 'Responsive environments' [CANTRELL] - represents another much expanded definition of 'computer aided design'. Algorithmic design that combines computer-based sensing, acting, and custom written code creating landscapes, buildings, and even cities epitomizes the impact of computation on Landscape architecture, as seen and imagined in the various examples throughout this volume.

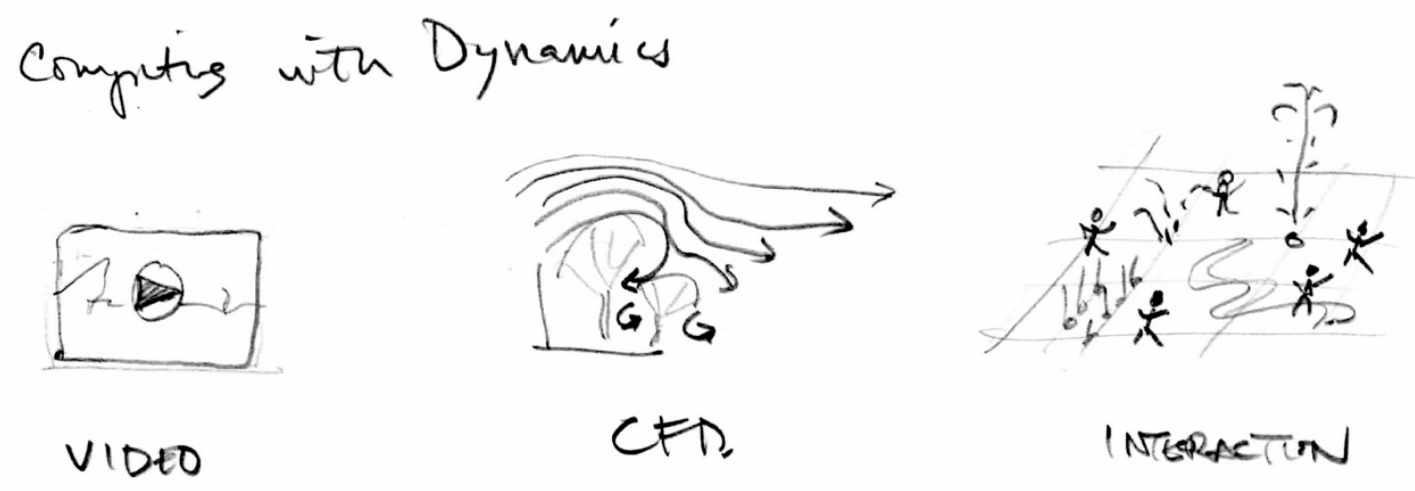

Figure 6. REPRESENTING DYNAMICS

\section{Algorithmic design}

As suggested by Turing's definition of the 'Universal Machine', that can perform any algorithmic process suitably described, computer-aided landscape architectural design can produce a wide variety of forms and landscapes, both analog and virtual - theoretically, any that can be suitably described. Rather than try to find a theoretical or ideological limit to the expressive/descriptive power of algorithms, let us consider a range of approaches, by way of illustrating a broad universe of possibilities. This list is neither exhaustive nor derived from any theory, but serves to illustrate a range of approaches, from simpler to more complex.

4.1 Pattern generation by repetition and variation

Perhaps the first industrial example of a pattern producing programmable machine was the Jacquard loom. Invented around 1801, this was a mechanical way of encoding complex woven patterns in a series of holes punched in cards, that were used to mechanically control the operation of individual warp- and weft-lifting hooks in the manufacture of textiles. The simplest woven fabric is just one color of thread and an 'over-under' pattern repeated in both axes. Adding multi-colored threads, and varying the pattern of over- and under- can generate geometric patterns in the fabric, as seen in brocades, plaids, etc. These can be made to look floral, or abstract, etc. - any pattern that can be described as a repeating arrangement of colored dots (in a 'raster') can be made to appear in the fabric. Similarly, just by the use of repetition and conditionals, in logical structures, a digital computer can do the same, and can generate either raster graphics, or vector (line-based) representations of grids, now often called 'warped grids', since they need not be consistently or simply rectangular. The use of grids (1-d, in a line, or 2-d, in an array, or even 3D, in a lattice) are pervasive in landscape design; from paving patterns, to planting plans and vegetative structures, to lighting, furniture and other objects. Simple mathematical measurements, counts, and ratios may in the landscape become interpreted as 'rhythmic', or 'repetitive', or given other qualitative experiential and design-intent-related descriptors. 

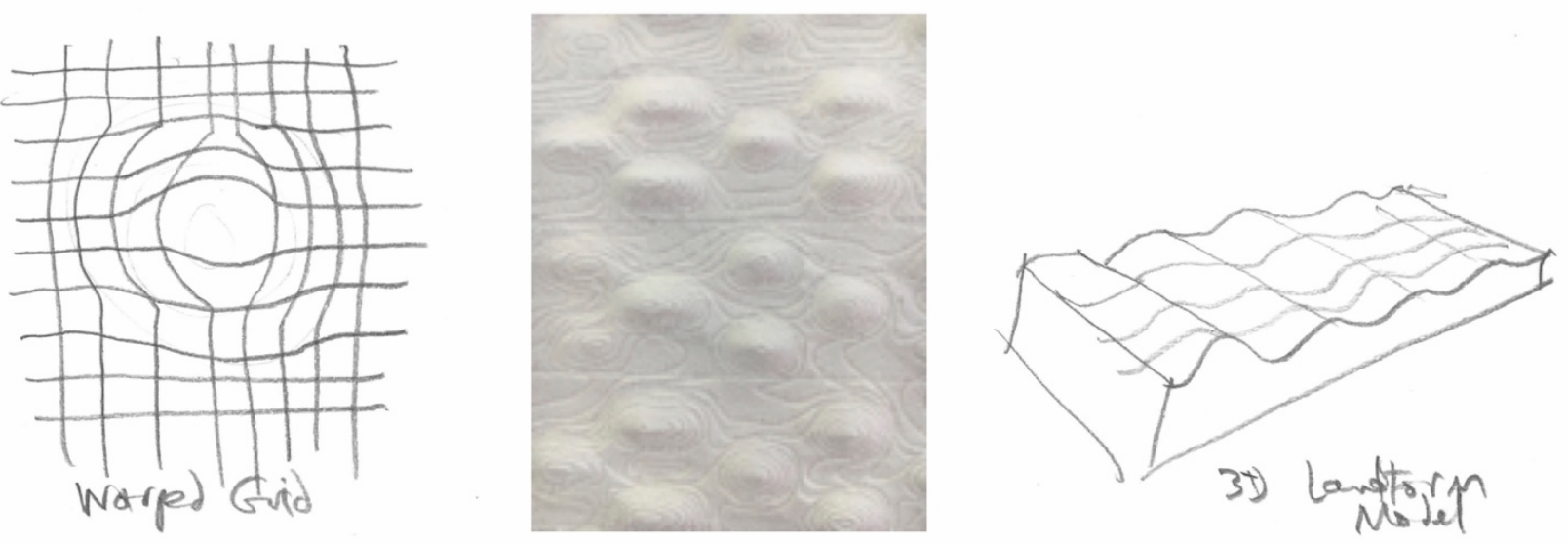

Figure 7: Warped Grids to 3D Landform

\subsection{Parametric generation}

A parameter is just a number, often one of several, that can define the state or behavior of a system (or, in computer coding, a value given as input into a procedure; other resulting values or behavior are determined by this parameter.) Usually, other values are also determined by some relationship, logical, mathematical, or other, to the parameter; and parameters are chosen to be critical or characteristic of the system being described or computed. So for a road, a key parameter might be 'width' (expressed in meters, or in lanes, etc.); and this parameter will not only cause a change in one dimension, but in other values as well. (Paving material, structural systems, planting details, curve radii, maximum slopes, and may other details may be determined by width.) The ability to write code that changes many values as the result of one input parameter makes possible 'parametric exploration' and 'parametric generation'. In some cases, a single 'slider' may be used to set a value somewhere between a predetermined minimum and maximum value; changing the slider slightly can sometimes generate a wide variety of changes in the result. In other parametric systems there may be number of parameters, that are separate, not determined by any other, and serve as the key defining input variables that distinguish the results. So code for parametric variation may be understood as a 'generating' system for a whole family of related designs, that all contain similar elements and relationships; these may be used as input a separate algorithmic 'testing' process, or perhaps just evaluated, by eye or intuition, by a human judge.

The powerful ability and attraction of writing algorithmic parametric design code is the ability to customize a generic process for specific conditions, as with an overall width, or density, or height restriction, etc.; and also to build in logic for responding to unique site conditions, so that different values and logic can be embedded at potentially every location, (however defined, e.g. location on a grid) throughout a site. This enables the hybridization of site-specific response with algorithmic form, with a potentially infinite range of variability, based on user input input, random variation, site conditions, etc. With the incorporation of sensors and actuators, then the design can itself be continually responsive to varying conditions (weather, occupancy, and other local- or remote- observations. Imagine a garden/installation that uses sensors and computer controlled irrigation and fans to mirror the rainfall and wind conditions of some distant locale, for example...)

\subsection{Fractal Recursion}

A particular form of repetition and variation that gives rise to intriguing pattern is the 'fractal' geometry [MANDELBROT] produced by a process of 'recursion', an algorithmic approach in which the output of a process is fed back into the same process, but with some regular variation (e.g. larger, or smaller, or with a change in angle) each time. Mathematically this gives rise to something called the 'Julia Set', and fractal formulas have been used to model the structure of many natural forms, such as coastlines, leaf edges, tree structures, stream networks, and others. Especially when a small amount of randomness is introduced, mimicking the natural worlds of variation, these patterns can become extremely life-like.

\subsection{Rule Based generation - Shape grammars \& L-systems}


In most algorithmic coding, there is a very clear but potentially complex sequence of operations, from beginning to end (with repetition and branching as required.) In rule-based programming, by contrast, there is a very simple structure: a set of rules, expressed as if-then conditions, some starting condition, and a simple sequence: at each time step, check through all rules, to see if any conditions for any rule have been meet, and if so apply that rule, potentially transforming the original condition; and repeat, until no more rules are firing. This approach, developed originally for attempting to encode rule of behavior for complex tasks, that might enable robotic performance, for example, can also be applied to graphical and 3-dimensional conditions. The so-called 'Shape Grammars' and 'L-systems' are algorithmic ways of generating shapes and forms buy the repeated application of spatial rules. These have been applied to the generation of buildings and gardens in plan [STINY], and to the generation of biologically inspired plant and animal forms [PRUSINKIEWICZ]

\subsection{Agent-based Simulations}

Carrying the 'object-oriented' paradigm a step further gives rise to the 'Agent-based' approach, in which objects represent animated 'beings' that have both attributes and relationships to other objects, but are imbued with behavior, and a 'point of view', or motivation, and are usually considered autonomous and mobile. Thus, software 'agents' representing shoppers in a commercial marketplace, or hikers or wildlife species in a wilderness landscape, can be 'let loose' in a simulated 3D environment, or terrain, and based on their programmed behavior, will interact with the landscape and each other, leaving an electronic trail of locations and corresponding attributes. This trail can then be analyzed to discover likely patterns of behavior, preferred paths, bottlenecks, and other characteristics of the designed landscape.

\subsection{Cellular automata, Particle Systems \& Swarms}

Finally, carrying agent-based approach a bit further, the agents may be relatively simple in their individual make-up, but when deployed in sufficient numbers - thousands, or tens-of-thousands, e.g. can constitute a system whose overall emergent behavior and resulting forms can be informative. An 'automaton' is a term originally applied to a physical mechanical device that could be programmed to produce repetitive simple behavior, switching between two or more states based on some inputs. A 'cellular automaton' ('CA') is usually a relatively simple bit of code, that can take several inputs and based on those inputs take on one of several states (on/off, or red/blue/green/, etc.); and usually deployed in a regular 2D grid where each cellular automaton is connected to all of its eight neighbors; at each step of a time clock, each CA takes all of its neighbors' states and input, potentially changes its own state as a result; and this proceeds through multiple time steps. Conway's 'Game of Life' [ConWAY] is a well-known example of this kind of logical system. CA models have been used to simulate the growth of settlements over time, with each CA representing patch of land, with states of development proceeding over time according to certain rules of individual behavior, which together in interaction end up creating a landscape as a mosaic of states, or land-uses [CA].

Very numerous CA systems have been used to analyze and model 'swarm' behavior, in which emergent properties of structure, motion, and apparent global intelligence appear from a large enough number of connected very-simple agents, much like a school of fish, or flock of birds [SWARM]. If the CA agents are freed from the 2D plane, and simulated in 3D space, they may be called a 'particle system'. Each particle in such a system is coded with 3D location, and other parameters such as direction, speed, size, etc., and these are programmed to change over time following some algorithmic rules. These algorithms may simulate physical processes like gravity, wind-resistance, erosion, flames, or smoke, and the resulting trajectories of the interacting particles give to rise to $2 \mathrm{D}$ and $3 \mathrm{D}$ forms representing a waterfall, or wave, fireball or cloud, etc. These techniques are extensively used in science and for film special effects; their role in landscape design is relatively unexplored, but intriguing.

As alluded to earlier, cellular automata and particle systems are examples of computational approaches that can benefit from parallel computing; each CA or particle can be computed by one processor in an array of thousands of processors, and simulations on relatively large arrays can be computed in relatively less time in a parallel computing environment than otherwise, making larger and more complex landscape simulations possible. Parallel processing in landscape architecture is not wellknown, -applied, or -studied, but the potential is obvious.

\subsection{Big data, machine learning}


All of the design generation methods described above assume that the steps of the recipe are written in code, and are applied unchanged unless the human designer/author changes the code. But modern computer scientists have become very interested in the proposition that with a sufficiently general approach, and access to large amounts of memory, computer programs can be written that both can learn from experience, and from training samples, and can over time self-modify, in effect re-writing the code they run. This is an advanced topic in Al research, but an increasingly well-reported one, and the potential for self-modifying code in the hands of computational designers is not far off. One factor driving this development is the increasing availability of very large data sets, collected by sensors all over the world and gathered from all across the internet. This 'big data' [BIGDATA] can represent hundreds of thousands of samples and observations, which can be analyzed and categorized by software, and form the basis for 'machine learning'. This development raises the possibility that computer-code can enter mote and more fully into the complete 'generate' and 'test' cycle; it is not too futuristic to imagine a computer program that could be set to monitoring global environmental conditions, at many scales, and that could begin to make design proposals, for improved conditions, at many scales and many locations, based on correlations and observations across a sufficiently large global sample. This raises the specter of 'computer-initiated design', not just computer-aided design ... and of the logical extreme case of 'Turing landscape' : landscapes designed with computation, with embedded computation, and by computation.

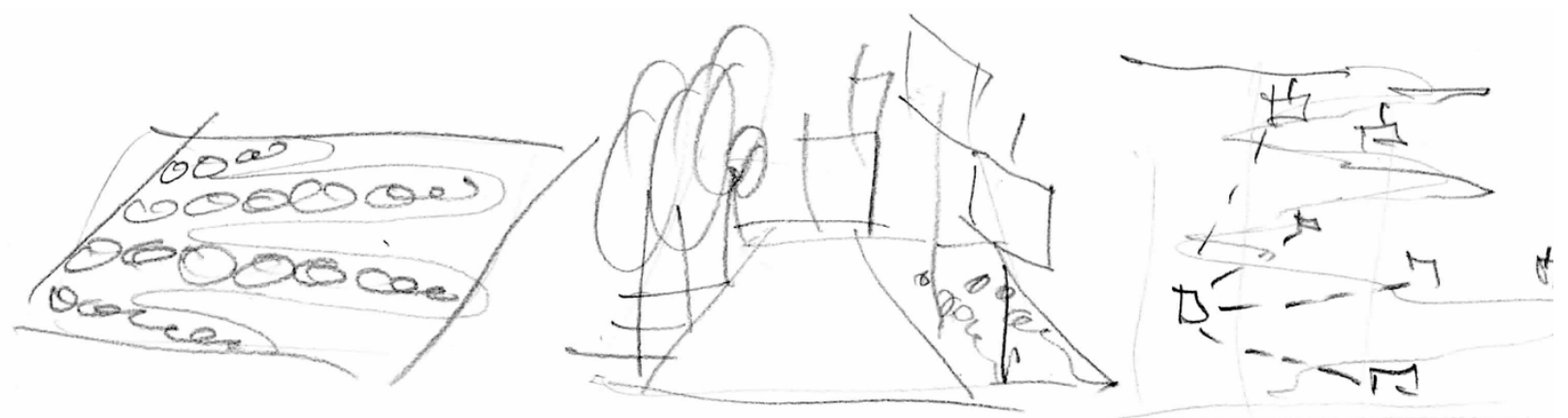

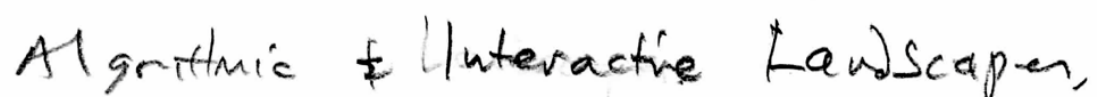

Figure 8. Algorithmic and Interactive landscapes

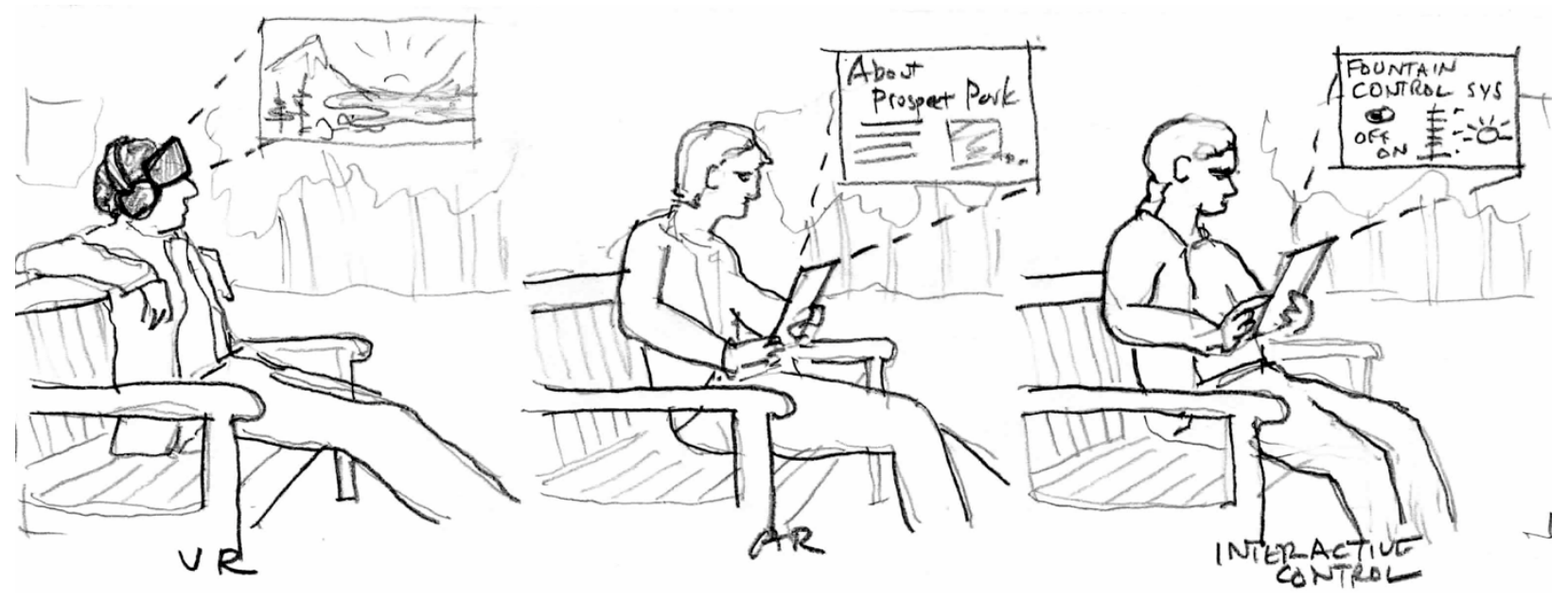

Figure 9. Three ways of being in a park with a computer...

\section{Turing landscapes}

In 1950, theorist and inventor Alan Turing, realizing that a 'Universal Computing machine' such as he had begun developing could produce output in the form of language (e.g. typed on a mechanical typewriter), famously proposed a test of the question "Can machines think?": if a human being, in communication with such a device, could not reliably tell whether she was communicating with another human being, or with a machine, the answer would be "yes". [TURING] This he predicted would be true in 
not so many years. (It has not yet been unequivocally demonstrated; although his prediction from that same time that "Computers will be able to play great Chess" has been.)

From "Can machines think?" to "Can machines design?" is not a great leap. What are some equivalent tests for 'Turing Landscapes'?

The 'Turing test' ("mimic a human being, in conversation, well enough to fool one") applied to landscape architecture, and to design, might be understood in three successively more challenging versions:

Landscape Turing Test v.1 (Representation) "Does a picture of a landscape look so real a human being can't tell if it's computer generated?" - We are already there! Photorealism in computer graphics, even of complex scenes such as dew on moss in a rainforest, or sea-foam on a sandy beach, has been fairly well conquered by the special effects industry in Hollywood, and to some extent supported by developments in the military. It is no longer really feasible for most people to know if a digital scene has been simulated by computation or captured by a camera in the wild. An early approach to landscape visualization used carefully cropped photographs of real plants, digitally composited together to form a scene, whose underlying geometry was simple planes and cylinders, but because of the texture-mapped photographs was sufficiently realistic for applications such as flight simulators and video games; but would not stand up to close inspection, such as enabled by more advanced VR applications. More complex 3D geometry can be created with sufficient processing power and powerful graphics capability; mathematically defined algorithms for plant form (such as figure 4) coupled with botanically-correct plant growth simulations, including such effects as phototropism and phyllotaxis, augmented with advanced image processing and texture mapping, can produce startlingly life-like high resolution images.

Landscape Turing Test v.2 (Process) "Does a built landscape behave so well we can't tell if it is 'designed or 'natural ?" - We are almost there. Using lighting, irrigation, and ventilation systems, coupled with high sensitivity sensors and high-tech building materials and techniques, we can now create landscapes that rival natural ones. Of course we can't replace natural evolution of plants and animals, nor do we know if these synthetic landscapes could last hundreds of years. Working with all-natural materials, just relocated, re-arranged and possibly re-formed by pruning or chiseling, Olmsted landscapes, as defined earlier, already blur the distinction between 'designed' and 'natural', especially over time. How much more can this distinction be blurred, with digitally manufactured and enhanced materials, digitally aided engineering of landform and water features, and mechatronic engineering of digitally designed and controlled structures and fixtures of all sorts. These designed / digital artifacts may be obvious, highcontrast, stainless steel and plastic, and obviously synthetic; or they may be subtle, embedded, integrated and hidden, serving to direct and corral natural forces, water flows, plant growth, patterns of light and shade, and landscape forms.

Landscape Turing Test v.3 (Design thinking) "Can you tell if a design has been generated by a computer or a person?" - Simply looking at a single instance, drawing, or model it would be very hard indeed. Many landscape designs can be characterized by a few simple paving and planting motifs (e.g. "double allee of trees (or of bagels!)", "checkerboard paving", "concentric rings"), and geometric patterns or mathematical ratios, which could in principle be programmed into a 'design machine'. How such a machine would respond to context and unique site conditions would be an immediate issue, where algorithmic approaches might fail, for there is little consensus on any rules or even general principles ("fit in" vs "stand out", e.g.) that might really distinguish a human designer versus a programmed response. In an extended design conversation - in which we would expect a human designer to know about human psychology and behavior as well as material properties and conceptual design ideas, likely a computer program today would still be identifiable by a certain lack of knowledge of these matters, and a lack of human-ness in its responses. But these requirements go back to the original Turing test - and in large part depend on a sufficiently large repertoire of human conversational gambits, and an encyclopedia of design knowledge and precedents. Current Al research into the topic of 'deep machine learning' may hold promise for enabling a computer to model high-level design abstractions, and so engage in a design conversation... But no such example has yet been developed or demonstrated, and there is currently little economic or other incentive to pursue this goal - there are so many other pressing areas where computer-based intelligence may fruitfully be applied (autonomous vehicles, smart energy systems, medical diagnosis, and others). And digital landscape architecture is truly still in its infancy; there is still 
very much to be discovered about the kind and qualities of landscapes made possible just by the combination of human designers, computer code, and electromechanical sensors and actuators.

In a less competitive approach than a 'test', thinking about the potential and characteristics of Turing landscapes is intriguing. The hybridization of human-machine, and analog-digital, at each of several scales in the landscape - from regional and climatic ('ecto), through local/site ('meso), to human body scale ('endo) - will bring all new kinds of landscapes and landscape experiences:

- Sensors at the regional scale that respond to weather, rainfall and wind conditions seasonally and daily, and communicate with actuators at at the local scale, to adjust dams, gates, sunshades, and windscreens to promote comfort and safety and deter erosion and plant damage; and deliver information, messages and images to wearable digital devices on your body, in your autonomous vehicle, or in your knapsack, or tent, or café table;

- Geodesign software systems that enable landscape architects and collaborating disciplines to design interventions from wildlife reserves to parks, housing developments, power stations, transportation hubs and individual buildings and courtyards, with integration of simulation tools that aid in identifying impacts (energy costs, carbon footprint, socio-economic costs, crowding, visual impacts, gain/loss of biodiversity and habitat, and others) and in information-enabled generate-test cycles design ever more fitting and carefully adjusted designs, proposals and projects;

- Computer controlled fabrication and GPS-enabled construction tools on site that enable precise site measurements and locations, material placement, for increased cost-effectiveness and adherence to precise design specifications;

- Digitally-augmented materials that can provide sensors, information, lighting, sound and other effects under interactive algorithmic control, such as pavement materials that provide lighting and navigational signage, hand railings that sense individuals' grips to respond and communicate, overhead adjustable canopies that provide shade and rain protection, lighting and informational graphics interacting with both regional weather sensors and individuals' digital devices and control systems...;

- Wearable digital devices providing location-enabled, customized navigation, access to information and entertainment to augment human experiences, from solitary to collaborative.

These possibilities, and others as yet unimagined, make it clear that the six traditional elements of Olmsted landscapes (landform, vegetation, water, infrastructure, animals, and atmosphere), the conception of the 'landscape experience', associated design practices and conventions, and even of 'landscape' itself, will necessarily be redefined in this Turing landscape era. All of the six essential elements of landscape are transformed by digital technologies and the possibilities of coding, in different ways to different degrees. Briefly:

\section{Turing Landscape Elements}

\section{Landform:}

Sensors embedded in the earth are already used to inform seismic, geological, hydrologic and agricultural processes. Autonomous bulldozers with only digital input and control are already in use in extractive and construction industries. Movable dykes, walls and barriers at the landform scale are already an element of flood-control in some environments; in the coming era of sea-level rise many new such constructions will be doubtless be required, coupled with complex sensor and control networks. Paving in roads and paths can have traffic and activity sensors embedded; new paving materials allow for integral lighting, digital signage and interactive way-finding aids directly embedded.

Water:

Sensors of water level, quality, velocity and temperature are already a part of hydrologic engineering for flood control, recreational, municipal and agricultural processes. Digitally controlled irrigation systems and fountains and waterworks in public open spaces are already part of the designer's palette. As water from surface channels, underground springs, residential waste systems, and rainfall on roofs and runoff from surrounding mountains and fields is better integrated into ecological and urban networks, enabling better aquifer recharge, and reduced waste, pollution, and erosion, the demands and opportunities for intelligent hydraulic control systems, designed for both beauty and function, will only increase, especially in the many water-stressed areas of the planet. 
Vegetation:

Plants are one area where it is least easy to imagine the hybridization of analog and digital materials and techniques: What exactly might a 'cyborg tree' or forest look like? Still, digitally managed irrigation systems for horticulture are commonplace; as are ornamentation and illumination schemes involving plants. Perhaps a structure providing bird habitat, with digitally controlled overhead shading devices and solar panels might be a new kind of 'Turing tree' in the landscape...?

Infrastructure:

Algorithmically designed smart buildings with sensors, controls, and actuators (automatic doors and elevators and windows, e.g.) are already commonplace. In the landscape, hardscape elements (paving) and furniture (benches, lights, etc.) offer the greatest opportunity for new hybridized digital forms. Sidewalks with embedded lighting, sensors and information channels are already appearing in modern environments. More multipurpose furniture incorporating WiFi networks and many kinds of sensors are also appearing. These elements, coupled with weather control innovations, will continue to help blur the distinction between inside (building) and outside (landscape) as landscape architecture, architecture, and urban design continue to co-evolve.

Animals:

Like plants, because they are also living, animals (including humans) are the least easy of all the elements to imagine as hybridized analog / digital beings (cyborgs). And yet, arguably, the modern landscape architect, and modern park/garden visitor, already come digitally augmented, with CAD/GIS software and laser surveying tools on the hand, and portable telecommunication devices, display systems, and personal sensors on the other. Landscape ecologists and wildlife manages are learning that animals outfitted with simple lightweight sensors can perform important functions as sensor agents in the landscape, and the opportunities for systems design incorporating sensor-bearing animals in public parks are fascinating...

Atmosphere:

Landscape architects have long had the opportunity to design with 'landscape lighting', but these designs have for the most part been rather simple, and their controls mostly limited to 'on-at-dusk, off-atdawn'. In an environment with multiple sources of sensor input, including presence and activities of people, other activities, and ambient weather conditions, how much more complex, expressive, and potentially informative can these become? As controllable multi-hue LEDs make possible all the colors of the rainbow for no extra expense, and LED/LCD display technologies broadly expand the range of shapes, sizes and structural possibilities for illumination systems, including embedded lighting in many kinds of materials, the possibilities for integrated control of atmospheric lighting - and indeed, other water-based and sonic- dimensions of atmosphere, as well - are much expanded for landscape architecture. Climate and seasonal effects also have great impact on landscape perception, use, and enjoyment, and climate data and sensors are now regularly a part of the landscape designer's toolkit.

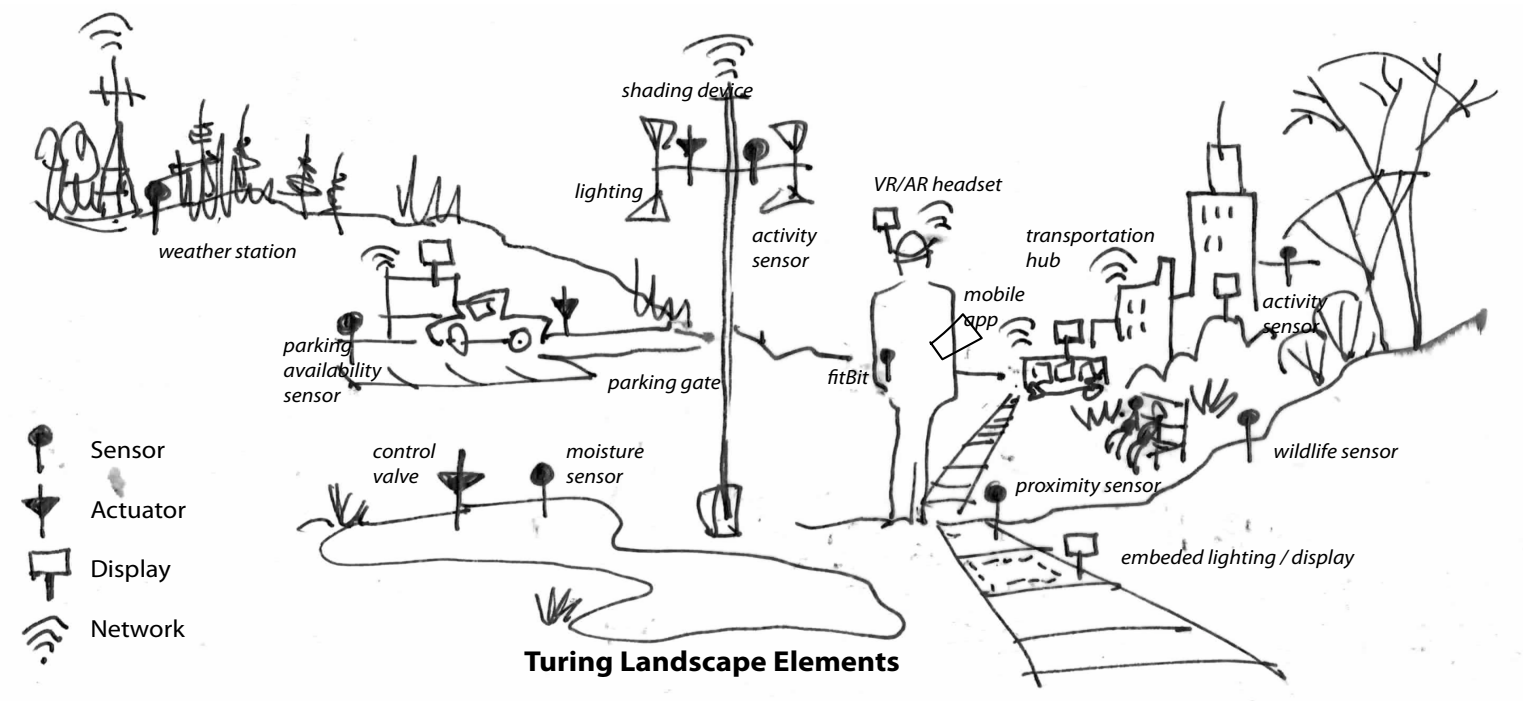


Figure 10. Turing landscape Elements in a Turing landscape

\section{Conclusion}

Landscape architecture and landscape architects have three distinct modes of interaction with computation:

1. Using software - as a tool

for Automation: from numbers to Lines to Graphics / Objects

2. Writing code - as a language

for Algorithms: from Logic to Code to Forms / Landscapes

3. Engaging with the Internet of Things - as a medium

for Augmentation: from Static to Dynamic to Interactive / Alive?

And Turing Landscapes of the twenty first century have three salient characteristics:

1. Designed using software, algorithmic approaches, \& simulation

2. Incorporating embedded sensors, actuators, and digital / algorithmic control of electromechanical elements

3. Providing information between people and environment, in digitally mediated responsive interactions and behavior

Clearly digital computation and Information Technology \& Communications (ITC) tools can be instrumental amplifiers of human eyes - enabling us to see and capture wavelengths and time frames impossible for the un-aided eye; and hands - enabling us to achieve strength and precision and resistance to environmental conditions that would be impossible for un-aided human hands; and brains enabling us to perform rapid calculations, store and retrieve enormous amounts of information, and explore chains of reasoning and trees of consequences impossible for the un-aided human mind. These tools are in extensive use and of real value to designers. Satellites, digital image processing, laser printers, 3D fabrication tools, mobile devices, crowd-sourced data, complex simulations, searchable databases, and so on are all essential tools of the trade, enabled by ITC developments.

But coding and algorithmic thinking - the essential information processing components of computation - are in their own right a rich design language for exploration and expression of design ideas, providing as much value to meaning-and-value-laden experience and expression ("poesis") as to purely instrumental manipulation ("techne").

Like other media, computation and algorithmic thinking bring their own characteristics, constraints and affordances, ways of knowing, ways of doing, and aesthetics. Coding is a language and a representational medium, and coding is itself a kind of designing, requiring abstraction, naming, modularization, and generate-and-test methods ('de-bugging); code can reflect and respond to real-world conditions as inputs, as well as express abstract ideas and intents. Digital representations (data) and processes (algorithms) are as rich and productive media as analog ones (earth, water, wood, plants, metal, paint, etc.) are; in their ability to either mimic the characteristics and limitations of those analogs, or to transcend / transform them, digital/algorithmic/computational media are powerful design enablers.

These tools of code and embedded computational intelligence and behavior are particularly effective in representing, manipulating and manifesting dynamics and responsive interaction, as other works in this volume demonstrate. Such dynamic/interactive/responsive landscapes make possible many new kinds of landscapes, landscape experiences, and landscape design approaches.

Digital techniques can enable dynamics, plasticity, mutability and responsiveness of varying types, at different frequencies and with finer control in virtual landscapes than with analog / living materials (earth, water, plants, e.g.) in real landscapes, and so are of great value and interest to 
landscape architecture. This does not mean that a day spent in a VR-viewing tank experiencing a simulated ocean-side beach will be as rich and nourishing to human body and spirit as a day spent at a real, unpolluted beach - but the former may be as intellectually and even aesthetically rewarding as time spent in a museum or at a movie. There are of course deep human evolutionary bonds to the 'real/natural' landscape, and its primitive elements of landform, vegetation, water, and atmosphere - there may even be as-yet undiscovered psychological and health benefits from human exposure to and interaction with these natural elements - and landscape architects and others will surely continue to celebrate and enable these contacts with nature.

The great promise of Augmented Reality ('AR') is in the hybridization of these real environments (both 'natural' and 'built') with digital and information-based processes. With these new techniques, materials, and technologies, landscape architects can fully embrace the potential for people to be not just 'visitors' in a landscape, but 'participants' in it.; and buildings and other mechanical infrastructure to be not just static / dead elements in the landscape, but dynamic, responsive, interactive (and 'alive'?) elements of the landscape design palette.

Although the intellectual challenge of $\mathrm{Al}$ and imagining and devising autonomous design algorithms and agents with artificial design intelligence (ADI) and capable of passing the 'Design Thinking' variant of the Turing test, as described above, will remain a fertile area of research and development into the indefinite future, for now the real challenge is to deeply explore and develop a complex and productive hybridization and collaboration between algorithmic/computational approaches - as tools, as language, and as medium - and human designers.

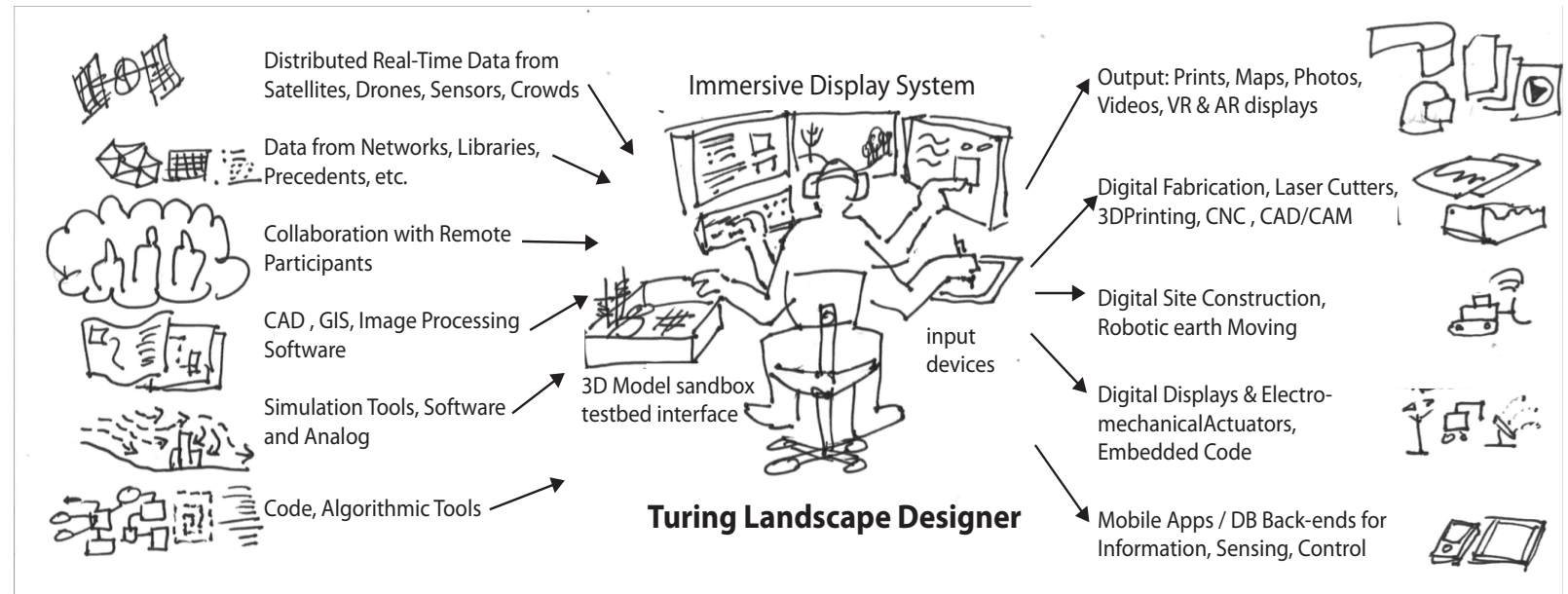

Figure 11. Turing Landscape Designer 
References

[1.] Leo Marx Machine in the Garden

[2.] Turing

[3.] Von Neumann

[4.] MacDougall Microcomputers in Landscape Architecture

[5.] Sutherland SketchPad

[6.] Processing Language

[7.] Geodesign Steinitz

[8.] Christopher Alexander

[9.] Herbert Simon Sciences of the Artificial

[10.] Rhinoceros \& Grasshopper McNeel

[11.] CFD software

[12.] Arduino www.arduino.cc

[13.] LittleBits www.littlebits.cc

[14.] Particle www.particle.io/prototype

[15.] Cantrell Responsive Environments

[16.] Fractal Mandelbrot

[17.] Stiny Shape Grammars

[18.] Prusinkiewicz Algorithmic Beauty of Plants

[19.] Conway's 'Game of Life'

[20.] Cellular Automata CA

[21.] Swarm

[22.] Turing Test

Ervin \& Hasbrouck,

Processing

BigData 